\title{
A summary of infinite element formulations for exterior Helmholtz problems
}

\section{Report}

Author(s):

Gerdes, K.

Publication date:

1997-08

Permanent link:

https://doi.org/10.3929/ethz-a-004284858

Rights / license:

In Copyright - Non-Commercial Use Permitted

Originally published in:

SAM Research Report 1997-11 


\title{
A summary of infinite element formulations for exterior Helmholtz problems
}

\author{
K. Gerdes
}

Research Report No. 97-11

August 1997

Seminar für Angewandte Mathematik

Eidgenössische Technische Hochschule

CH-8092 Zürich

Switzerland 


\title{
A summary of infinite element formulations for exterior Helmholtz problems
}

\author{
K. Gerdes \\ Seminar für Angewandte Mathematik \\ Eidgenössische Technische Hochschule \\ CH-8092 Zürich \\ Switzerland
}

Research Report No. 97-11_ August 1997

\begin{abstract}
This work is devoted to a study and summary of different Infinite Element (IE) formulations for Helmholtz problems in arbitrary exterior domains. The theoretical setting for each of the different formulations is presented and related to the mathematical existence theory. The influences of a bilinear or a sesquilinear formulation are discussed as well as possible extensions to other elements. The implementation of the Infinite Element Method (IEM) incorporates the use of 2D and $3 \mathrm{D} h p$ Finite Elements and allows for $h p$-adaptive refinements. Numerical results show the computational efficiency of the coupled Finite-Infinite Element methodology.
\end{abstract}




\section{Introduction}

The present paper is motivated by the new concept on various infinite elements by Burnett [4], Astley et all [1], Cremers et all [5, 6] and our own work [7, 11, 12, 13]. These new IEM are extensions of the original IEM, which was introduced by Bettess [3].

The problem of interest deals with scattering of acoustic waves on elastic or rigid objects. The mathematical formulation consists of the Helmholtz equation in the exterior domain accompanied by the Sommerfeld radiation condition and Neumann boundary condition on the boundary of the scatterer (rigid scattering).

Problems of the described type are usually solved using various versions of the Boundary Element Method (BEM) [9]. The mathematics of the BE approximation (especially in the Galerkin version) is well established and the method delivers reliable results in the whole range of wave numbers. The main drawback of the BEM is its cost - the method becomes prohibitively expensive for large wave numbers. The approach based on the truncation of the infinite domain to a finite one and application of the so called absorbing boundary conditions has always been an alternative technique to solve the problem [14, 15]. Another technique is to approximate the Dirichlet-to-Neumann operator by solving an auxiliary Riccati equation [17]. The drawback is that this method and the exact absorbing boundary conditions correspond to nonlocal operators and result in dense matrices. The local absorbing boundary conditions are not exact but are computationally attractive. The recent versions of the Infinite Elements that are based on multipole expansions fit into the local absorbing boundary condition framework and offer accuracy of arbitrary high order and can be coupled with standard $C^{0}$ finite elements. The recent results on convergence of such methods [2, 7, 8] support reliability of such an approach and add to its attractiveness. An essential difference between the various versions of the infinite elements has been recently pointed out in $[2,13]$. The difference lies in the fact whether one does or does not use the complex conjugate over the test functions (sesquilinear vs. bilinear form formulation).

In this paper, we investigate the Galerkin FE/IE formulations for a three dimensional rigid scattering problem. The FEM is applied in a finite annular domain obtained by the domain decomposition approach in the exterior of the scatterer. The scatterer is first enclosed by a sphere of finite radius, forming an artificial boundary inside the acoustic medium. In the domain between the scatterer and the sphere, a FEM is used for the numerical solution whereas outside the artificial boundary the solution is approximated with an IEM. This procedure has been described in detail, together with a convergence analysis, for the special case of a spherical scatterer $[7,11,12,13]$. Here, we account for a general shape of the scatterer, which is located within the unit sphere. In particular, we study the different variational formulations that can be obtained by using a bilinear form or a sesquilinear form in the weak formulation. This corresponds to using or not using the complex conjugate in the weak formulation [13]. Additionally, we present the 
different space settings, that were introduced by Leis [16] and in [7] for the Burnett formulation. These possible space settings result in different convergence properties of the IE schemes.

The plan of the presentation is as follows. We begin by formulating the exterior Helmholtz problem in section 2. The different coupled FE/IE methodologies are presented in section 3. Aspects regarding the numerical implementation and numerical experiments are presented in section 4 . We finish the presentation with concluding remarks in section 5 .

\section{The exterior Helmholtz problem}

We first introduce the notation and the classical formulation of the exterior Helmholtz problem.

\subsection{Notation}

- $\Omega \in \mathbb{R}^{3}$ is a domain occupied by the rigid scatterer and contained in the unit sphere

- $\Omega^{e}=\mathbb{R}^{3}-\Omega$ is the domain exterior to the scatterer

- $\Gamma_{s}=\left\{\boldsymbol{x} \in \mathbb{R}^{3} ;|\boldsymbol{x}|=1\right\}$ is the surface of the unit sphere

- $\Omega_{s}^{e}=\left\{\boldsymbol{x} \in \mathbb{R}^{3} ;|\boldsymbol{x}|>1\right\}$ is the domain exterior to the unit sphere

- $\Gamma=\partial \Omega$ is the surface of the rigid scatterer

- $\Omega_{s}=\left\{\boldsymbol{x} \in \mathbb{R}^{3} ;|\boldsymbol{x}| \leq 1\right\}-\Omega$ is the domain between the unit sphere and the rigid scatterer

The notation is illustrated in Figure 1 and we emphasize again that we assume without loss of generality that the scatterer is located within the unit sphere.

\subsection{Classical Formulation of the Problem}

The goal is to find a function $u=u(\boldsymbol{x})$ satisfying:

- the Helmholtz equation in the domain exterior to the scatterer,

$$
-\Delta u-k^{2} u=0 \quad \text { in } \quad \Omega^{e}
$$

where $k$ is the wave number; 


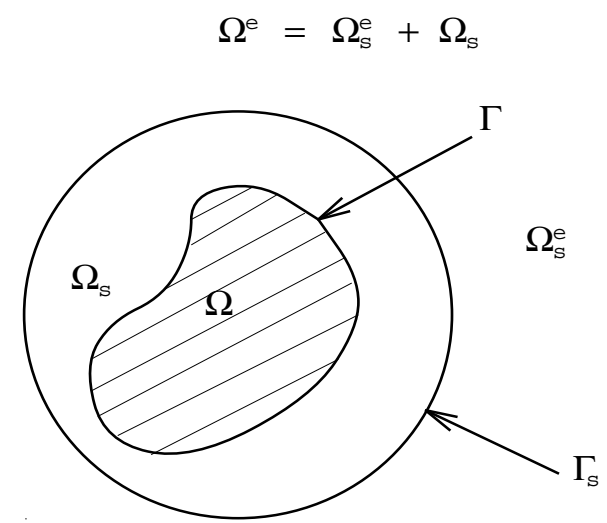

Figure 1: Notation. Scatterer within a unit sphere.

- a Neumann boundary condition on the scatterer

$$
\nabla_{n} u=g \quad \text { for } \quad \boldsymbol{x} \in \Gamma
$$

where $g$ is a prescribed function on $\Gamma$;

- the Sommerfeld radiation condition at infinity,

$$
\left|\frac{\partial u}{\partial n}-i k u\right|=O\left(\frac{1}{r^{2}}\right) .
$$

\section{The coupled $h p$ FE/IE methodology}

The coupled FE/IE methodology is introduced and analyzed in the following.

\subsection{Variational formulations for general exterior domains}

The various variational formulations are derived by starting with a given "truncated" exterior domain $\Omega_{\gamma}^{e}$,

$$
\Omega_{\gamma}^{e}=\Omega^{e} \cap\left\{\boldsymbol{x} \in \mathbb{R}^{3}:|\boldsymbol{x}|<\gamma\right\} .
$$

The domain $\Omega_{\gamma}^{e}$ is a finite subset of $\Omega^{e}$ and we note that the truncation occurs by a spherical surface with a possibly large radius $\gamma$. Obviously, $\Omega_{\gamma}^{e}$ converges to $\Omega^{e}$ for $\gamma$ approaching infinity. The shape of the scatterer does not need to be spherical. Thus, the following derivation applies to general scatterers.

The Helmholtz equation is multiplied by a test function $v$ and then integrated over $\Omega^{e}$. This will clearly lead to a bilinear form. We note that the Helmholtz equation can 
also be multiplied by the complex conjugate of a test function $v$, i.e. $\bar{v}$, and then be integrated. This results in a sesquilinear form after performing the integration by parts. The Neumann boundary condition (2.2) contributes with a boundary integral to the right hand side and for the bilinear and sesquilinear case we obtain respectively

$$
\begin{aligned}
& \int_{\Omega_{\gamma}^{e}} \nabla u \cdot \nabla v d \Omega_{\gamma}^{e}-k^{2} \int_{\Omega_{\gamma}^{e}} u v d \Omega_{\gamma}^{e}-\int_{S_{\gamma}} \frac{\partial u}{\partial n} v d S_{\gamma}=\int_{\partial \Omega^{e}} g v d S, \\
& \int_{\Omega_{\gamma}^{e}} \nabla u \cdot \nabla \bar{v} d \Omega_{\gamma}^{e}-k^{2} \int_{\Omega_{\gamma}^{e}} u \bar{v} d \Omega_{\gamma}^{e}-\int_{S_{\gamma}} \frac{\partial u}{\partial n} \bar{v} d S_{\gamma}=\int_{\partial \Omega^{e}} g \bar{v} d S,
\end{aligned}
$$

where $v$ is any admissible test function and $S_{\gamma}$ is the "truncating" sphere with radius $r=\gamma$. The normal derivative of $u$ on $S_{\gamma}$ can be eliminated by applying the Sommerfeld radiation condition. Therefore, the Sommerfeld radiation condition (2.3) is written in the form

$$
\frac{\partial u}{\partial r}=i k u+\varphi(\boldsymbol{x})
$$

where $\varphi(\boldsymbol{x})=O\left(r^{-2}\right)$ is an unknown function. This is built into the variational formulation (3.1) and (3.2) by substituting (3.3) for $\partial u / \partial n=\partial u / \partial r$ in the corresponding boundary term formula. This leads to

$$
\begin{aligned}
& \int_{\Omega_{\gamma}^{e}} \nabla u \cdot \nabla v d \Omega_{\gamma}^{e}-k^{2} \int_{\Omega_{\gamma}^{e}} u v d \Omega_{\gamma}^{e}-i k \int_{S_{\gamma}} u v d S_{\gamma}=\int_{\partial \Omega^{e}} g v d S+\int_{S_{\gamma}} \varphi v d S_{\gamma}, \\
& \int_{\Omega_{\gamma}^{e}} \nabla u \cdot \nabla \bar{v} d \Omega_{\gamma}^{e}-k^{2} \int_{\Omega_{\gamma}^{e}} u \bar{v} d \Omega_{\gamma}^{e}-i k \int_{S_{\gamma}} u \bar{v} d S_{\gamma}=\int_{\partial \Omega^{e}} g \bar{v} d S+\int_{S_{\gamma}} \varphi \bar{v} d S_{\gamma} .
\end{aligned}
$$

Passing formally with $\gamma \rightarrow \infty$, we obtain for both cases

$$
\begin{aligned}
& \int_{\Omega^{e}} \nabla u \cdot \nabla v d \Omega^{e}-k^{2} \int_{\Omega^{e}} u v d \Omega^{e}-i k \lim _{\gamma \rightarrow \infty} \int_{S_{\gamma}} u v d S_{\gamma} \\
= & \int_{\Gamma} g v d S+\lim _{\gamma \rightarrow \infty} \int_{S_{\gamma}} \varphi v d S_{\gamma}, \\
& \int_{\Omega^{e}} \nabla u \cdot \nabla \bar{v} d \Omega^{e}-k^{2} \int_{\Omega^{e}} u \bar{v} d \Omega^{e}-i k \lim _{\gamma \rightarrow \infty} \int_{S_{\gamma}} u \bar{v} d S_{\gamma} \\
= & \int_{\Gamma} g \bar{v} d S+\lim _{\gamma \rightarrow \infty} \int_{S_{\gamma}} \varphi \bar{v} d S_{\gamma} .
\end{aligned}
$$

The general theory in [16], shows that the leading order term of $\left.u\right|_{\Omega_{s}^{e}}$ is of the form

$$
u_{0}(\boldsymbol{x}) \frac{\exp (i k r)}{r}, \quad \boldsymbol{x} \in \Gamma_{s},
$$

and, consequently, both $u$ and its gradient, $\nabla u$, are not $L^{2}$-integrable over the exterior domain. Therefore, it is necessary to enforce the integrability of the not $L^{2}$-integrable 
terms in the conjugated and unconjugated case. This problem can be resolved in two different ways. Either by defining appropriate weighted Sobolev spaces or by interpreting integrals in the Cauchy Principle Value sense. The first approach was introduced by Leis [16] and is based on a precise mathematical theory and was studied in $[1,5,6,11]$. The second approach was introduced by Burnett [4] and a corresponding mathematical theory does not exist yet. This Burnett formulation was also studied and compared to the Leis method on a spherical domain in [13]. We note that the integral involving the unknown function $\varphi$ in (3.6) and (3.7) vanishes in both approaches if $\gamma \rightarrow \infty$. Both methods are described in the following for general scatters.

The Leis method employs test functions of order $O\left(r^{-3}\right)$ in $\Omega_{s}^{e}$. This makes it possible to interprete the integrals in the usual Lebesgue sense. This particular choice of the test functions does not allow one to build the radiation condition into the weak formulation, and the Sommerfeld condition has to be included directly in the definition of the spaces. This leads to the definition of the following weighted Sobolev space

$$
H_{w}^{1}\left(\Omega^{e}\right)=\left\{u:\|u\|_{w}^{1}<\infty\right\}
$$

with the norm $\|u\|_{w}^{1}$ corresponding to the inner product

$$
(u, v)_{w}^{1}=\int_{\Omega^{e}} w u \bar{v}+w \nabla u \cdot \nabla \bar{v} d \Omega^{e}+\int_{\Omega^{e}}\left(\frac{\partial u}{\partial r}-i k u\right) \overline{\left(\frac{\partial v}{\partial r}-i k v\right)} d \Omega^{e} .
$$

Two particular weights are of interest,

$$
w(\boldsymbol{x})=\left\{\begin{array}{c}
1 \text { for } \quad r=|\boldsymbol{x}| \leq 1 \\
\frac{1}{r^{2}} \text { for } \quad r=|\boldsymbol{x}|>1
\end{array}\right.
$$

and a "dual" weight

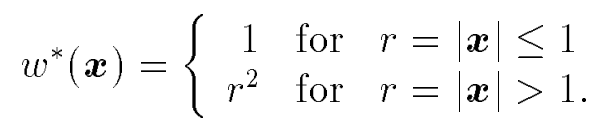

The variational formulation reads in the bilinear and sesquilinear case

$$
\begin{aligned}
& \left\{\begin{array}{l}
\text { Find } u \in H_{w}^{1}\left(\Omega^{e}\right) \text { such that } \\
\int_{\Omega^{e}} \nabla u \cdot \nabla v d \Omega^{e}-k^{2} \int_{\Omega^{e}} u v d \Omega^{e}=\int_{\partial \Omega^{e}} g v d S \quad \forall v \in H_{w^{*}}^{1}\left(\Omega^{e}\right)
\end{array}\right. \\
& \left\{\begin{array}{l}
\text { Find } u \in H_{w}^{1}\left(\Omega^{e}\right) \text { such that } \\
\int_{\Omega^{e}} \nabla u \cdot \nabla \bar{v} d \Omega^{e}-k^{2} \int_{\Omega^{e}} u \bar{v} d \Omega^{e}=\int_{\partial \Omega^{e}} g \bar{v} d S \quad \forall v \in H_{w^{*}}^{1}\left(\Omega^{e}\right)
\end{array}\right.
\end{aligned}
$$

\section{Remarks:}


1. The proposed variational formulation corresponds to an extension of the operator setting of Leis [16], where the domain of the operator is restricted to a subspace of $H_{w}^{1}\left(\Omega^{e}\right)$ consisting of all functions for which the (Helmholtz) operator value is in the weighted $L_{w^{*}}^{2}\left(\Omega^{e}\right)$ space. With these assumptions, Leis proves the uniqueness and existence of solutions, showing that the resulting operator is bounded below with a constant locally independent of the wave number $k$.

2. The Leis method is formally identical in the bilinear and sesquilinear formulation but the application of the complex conjugate renders the convergence properties of the Leis formulation, as was studied in $[2,13]$.

3. The integrals that correspond to the infinite elements can be computed more easily in the conjugated case, compare section 4.

The Burnett method [4] is based on the fact that both solution $u$ and test function $v$ are represented outside of the unit sphere in the form

$$
\begin{aligned}
u(r, \theta, \phi) & =\frac{\exp (i k r)}{r} u_{0}(\theta, \phi)+U(r, \theta, \phi), \\
v(r, \theta, \phi) & =\frac{\exp (i k r)}{r} v_{0}(\theta, \phi)+V(r, \theta, \phi),
\end{aligned}
$$

where $r, \theta, \phi$ are spherical coordinates. The functions $u_{0}(\theta, \phi)$ and $v_{0}(\theta, \phi)$ denote the radiation patterns, and functions $U(r, \theta, \phi), V(r, \theta, \phi)$ are from $H^{1}\left(\Omega_{s}^{e}\right)$, i.e. both $U, V$ and their gradients $\nabla U, \nabla V$ are square-integrable. Function $u$ of this form satisfies automatically the Sommerfeld radiation condition. Upon substituting formulas (3.12) into (3.1), (3.2) and cancelling out terms involving the radiation patterns, one can pass to the limit with $\gamma \rightarrow \infty$. Contrary to the weighted spaces formulation, the integral over $S_{\gamma}$ involving the radiation patterns will not vanish in the limit. In this case, the bilinear and sesquilinear formulation following the Burnett approach read as

$$
\begin{aligned}
& \left\{\begin{array}{l}
\text { Find } u \in H_{w}^{1}\left(\Omega^{e}\right) \text { such that } \\
\int_{\Omega^{e}} \nabla u \cdot \nabla v d \Omega^{e}-k^{2} \int_{\Omega^{e}} u v d \Omega^{e}-i k \lim _{\gamma \rightarrow \infty} \int_{S_{\gamma}} u v d S_{\gamma} \\
=\int_{\partial \Omega^{e}} g v d S \quad \forall v \in H_{w}^{1}\left(\Omega^{e}\right),
\end{array}\right. \\
& \left\{\begin{array}{l}
\text { Find } u \in H_{w}^{1}\left(\Omega^{e}\right) \text { such that } \\
\int_{\Omega^{e}} \nabla u \cdot \nabla \bar{v} d \Omega^{e}-k^{2} \int_{\Omega^{e}} u \bar{v} d \Omega^{e}-i k \lim _{\gamma \rightarrow \infty} \int_{S_{\gamma}} u \bar{v} d S_{\gamma} \\
=\int_{\partial \Omega^{e}} g \bar{v} d S \quad \forall v \in H_{w}^{1}\left(\Omega^{e}\right),
\end{array}\right.
\end{aligned}
$$


respectively. The integrands in formulations (3.13) and (3.14) are understood in the Cauchy Principle Value sense and we emphasize that precisely the integrands that are not $L^{2}$ integrable do cancel each other out. In particular, in the sesquilinear formulation there is a contribution of the additional surface integral that is present in the weak formulation, for details compare [13]. It is further evident that the space setting in the Burnett approach is symmetric, whereas the Leis approach employs a non-symmetric space setting and results in a non-symmetric global linear system, although the bilinear and sesquilinear forms are symmetric.

\subsection{Separation of Variables in $\Omega_{s}^{e}$}

For completeness we review shortly the results of the separation of variables procedure that motivate the IEM. For a detailed discussion see [11]. The form of the solution outside the unit sphere is:

$$
u(r, \theta, \phi)=\sum_{n=0}^{\infty} \sum_{m=0}^{n} h_{n}(k r) P_{n}^{m}(\cos \theta)\left(A_{n m} \cos (m \phi)+B_{n m} \sin (m \phi)\right)
$$

where $P_{n}^{m}(\cos \theta)$ are the Legendre functions and $h_{n}(k r)$ are the spherical Hankel functions of the first kind. It is clear that the coefficients $A_{n m}$ and $B_{n m}$ cannot be determined unless a boundary condition is given on $\Gamma_{s}$. We note that the spherical Hankel functions of the first kind can be represented by

$$
h_{n}(k r)=\sum_{m=0}^{n} \frac{\exp (i k r)}{r^{m+1}} \frac{\exp \left(-i \frac{\pi}{2}(n+1)\right)}{k(2 k)^{m}} i^{m}\left(n+\frac{1}{2}, m\right),
$$

with

$$
\left(n+\frac{1}{2}, m\right)= \begin{cases}1 & m=0 \\ \prod_{k=1}^{m}(n+k) \cdot \prod_{k=1}^{m} \frac{(n-m+k)}{k} & m \geq 1 .\end{cases}
$$

\subsection{Definition of the $h p$-infinite element}

The following definition of shape functions in the radial direction is motivated by the form of the solution outside the sphere, i.e. by (3.15) and (3.16):

the trial functions

$$
\psi_{j}(r)=\frac{\exp (i k r)}{r^{j}}, \quad j \geq 1
$$

the test functions

$$
\tilde{\psi}_{j}(r)=\frac{\exp (i k r)}{r^{j+m}}, \quad j \geq 1,
$$

with $m=2$ in the Leis formulation and $m=0$ in the Burnett formulation. 
Note in the Leis formulation the different powers of $r$ in the denominators. The use of the sesquilinear formulation does eliminate the necessity to integrate the oscillatory component $\exp (i k r)$, see section 4 .

The infinite element shape functions are then given as tensor products of $2 \mathrm{D} \mathrm{hp}$ finite element shape functions and the functions introduced in (3.17) and (3.18) respectively, i.e. a typical infinite element trial shape function $N_{l}(r, \boldsymbol{x})$ is given by

$$
N_{l}(r, \boldsymbol{x})=N_{l(i, j)}(r, \boldsymbol{x})=\psi_{j}(r) \cdot \varphi_{i}(\boldsymbol{x}), \quad r>1, \boldsymbol{x} \in \Gamma_{s} .
$$

In order to minimize the interaction between infinite elements and 3D $h p$ finite elements $\Omega_{s}$, the IE trial shape functions are now modified as follows

$$
N_{l(i, j)}(r, \boldsymbol{x})=\left\{\begin{array}{cl}
\exp (-i k) \psi_{j}(r) \varphi_{i}(\boldsymbol{x}) & j=1, r>1, \boldsymbol{x} \in \Gamma_{s} \\
\exp (-i k)\left(\psi_{j}(r)-\psi_{1}(r)\right) \varphi_{i}(\boldsymbol{x}) & j \geq 2, r>1, \boldsymbol{x} \in \Gamma_{s}
\end{array}\right.
$$

with an identical modification for the test functions. In this way, all the shape functions corresponding to $j \geq 2$ will contribute to basis functions with support outside of the unit sphere only. Inclusion of the exponential factor $\exp (-i k)$ in the new definition forces the infinite element shape functions to coincide with the standard $3 \mathrm{D} h p$ finite element shape functions on the surface of the sphere.

It is evident that this definition of IE shape functions applies to the Leis and Burnett formulation in the bilinear and sesquilinear form. Also, the definition is valid for other $2 \mathrm{D}$ and 3D master elements. In the following we use triangular prismatic elements in $\Omega_{s}$ and triangular elements on the surface of the unit sphere. It is also possible to apply quadrilateral elements on the surface of the unit sphere and cubic elements in $\Omega_{s}$. A combination of triangular and tetrahedral elements is also possible. The different choices of master elements only affect the definition of functions $\varphi_{i}$ in (3.20) but otherwise the IE shape functions $N_{l(i, j)}$ remain the same.

\section{$3.4 h p$-adaptive discretization}

The domain $\Omega_{s}$ in between the scatterer and the truncating sphere is discretized using triangular prismatic $h p$-elements. The faces of such elements are triangles and therefore the infinite elements are based on triangular 2D elements, which are extended towards infinity, compare Figure 2. The prismatic and triangular elements can be easily replaced by other elements, e.g. tetrahedral elements or cubic elements in combination with rectangular elements on the surface and we hope to report on such an extension in a forthcoming publication.

The prismatic $h p$ master element, shown in Figure 3, consists of six vertex nodes and fifteen higher-order nodes: nine mid-edge, two mid-base, three mid-side and one middle 


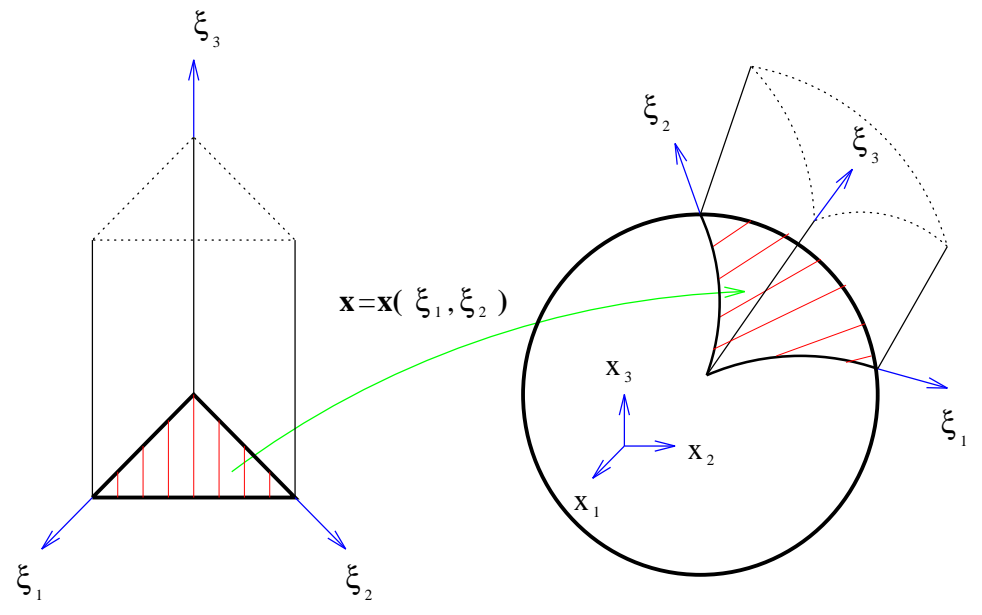

Figure 2: Infinite Element.

node. The corresponding shape functions are tensor products of the 2D triangle shape functions $\chi_{i}\left(\xi_{1}, \xi_{2}\right)$ discussed in [10] and 1D incremental shape functions, i.e. $\chi_{j}\left(\xi_{3}\right)$

$$
\chi_{k}\left(\xi_{1}, \xi_{2}, \xi_{3}\right)=\chi_{i}\left(\xi_{1}, \xi_{2}\right) \cdot \chi_{j}\left(\xi_{3}\right), \quad k=k(i, j) .
$$

For $j=1,2$, functions $\chi_{j}\left(\xi_{3}\right)$ are the regular linear shape functions. Given a particular order of approximation $q$ in the "vertical" $\left(\xi_{3}\right)$ direction, functions $\chi_{j}\left(\xi_{3}\right), j=3, \ldots, q+1$, coincide with the regular 1D Lagrange shape functions of order $q$, vanishing at the endpoints. Consequently, the mid-side and the middle node have two corresponding orders of approximations: a horizontal $p$ and a vertical order $q$. For that reason, we are able to have a variable approximation order within the element, which allows for a full $h p$-computation. The 2D $h p$ master triangle is defined in a similar way, compare Figure 3 , and also allows for a varying polynomial approximation order within the element. For all details concerning the definition of the master elements we refer to [10].

Figure 4 shows three quarters of a typical initial FE mesh for the scatterer being a sphere with radius 0.5 , which is inside the unit sphere. The infinite elements are not shown, but the mesh on the surface of the unit sphere is clearly visible and sufficient for the infinite element computations. In this example there are 2 layers of 3D finite elements with 24 elements per layer, angular polynomial approximation order $p=4$, radial polynomial approximation order $q=2$ and 24 elements on the surface of the unit sphere, which represent 24 infinite elements. 


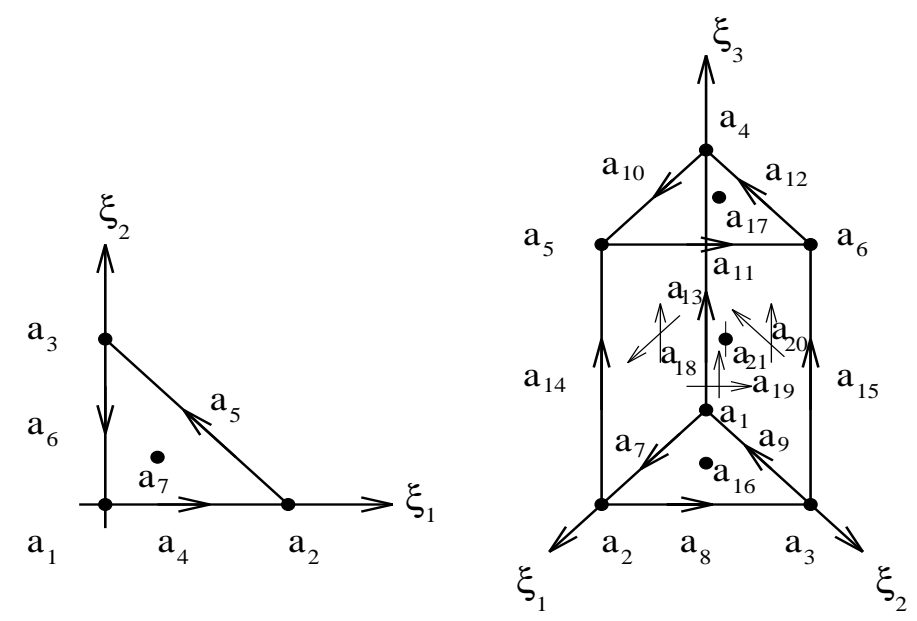

Figure 3: Master triangle and master prism

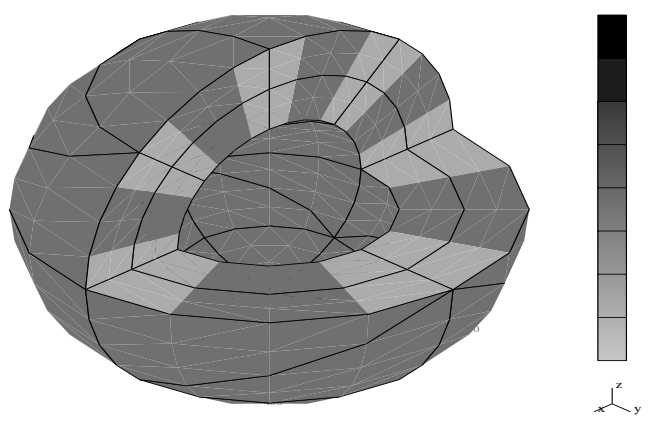

Figure 4: hp Finite/Infinite Element Mesh. Three quarters of the mesh are displayed. 


\section{Numerical Implementation}

\subsection{Element computations}

The implementation of the 3D finite elements is done in the standard way and the computations for the infinite elements are similar to 2D FE computations. The integration of the IE shape functions in the radial direction involves the following integrals in the conjugated formulations

$$
\int_{1}^{\infty} \frac{\exp (i k r) \exp (-i k r)}{r^{j}} d r=\frac{1}{j-1}, \quad j \geq 2
$$

and this is simply used in the element computations. In the unconjugated version terms of the form

$$
\int_{1}^{\infty} \frac{\exp (i k r) \exp (i k r)}{r^{j}} d r=\int_{1}^{\infty} \frac{\exp (2 i k r)}{r^{j}} d r
$$

do appear, which can be evaluated using the exponential integral. The following representation is used to obtain a form of the radial integral that can easily be integrated,

$$
\int_{1}^{\infty} \frac{\exp (2 i k r)}{r^{n}} d r=\sum_{l=1}^{n-1} \frac{-(-i 2 k)^{l-1} \exp (2 i k)}{\prod_{\hat{l}=1}^{l}-n+\hat{l}}+\frac{(-i 2 k)^{n-1}}{\prod_{\hat{l}=1}^{n-1}-n+\hat{l}} \int_{1}^{\infty} \frac{\exp (2 i k r)}{r} d r
$$

where $n \geq 1, \sum_{l=1}^{0}:=0$ and $\prod_{\hat{l}=1}^{0}:=1$. The representation (4.3) can be easily verified by induction. Having these integrals computed ahead of time, the calculation of the infinite element stiffness matrices is straightforward and reduces to standard 2D FE-like calculations. The element contributions are then assembled by a generalized assembling procedure [10], which is adapted to handle complex valued matrices and can also deal with general $h p$ meshes. The element load vector is calculated similarly. It should be noted that the final global system may not be symmetric.

\subsection{Scattering of a Plane Wave by a Rigid Sphere}

In [11] we derived the form of the scattered wave $p^{s}$ on a rigid sphere with radius 0.5 corresponding to an incident plane wave:

$$
p^{s}=\sum_{n=0}^{\infty} h_{n}(k r) P_{n}^{m}(\cos \theta) A_{n},
$$

with $A_{n}$ given by

$$
A_{n}=\frac{-\left.P_{i n c}(2 n+1) i^{n} \frac{\partial j_{n}(k r)}{\partial r}\right|_{r=0.5}}{\left.\frac{\partial h_{n}(k r)}{\partial r}\right|_{r=0.5}} \quad \forall n \geq 1
$$




\subsection{Error Calculations}

The weighted $H^{1}$-norm is consistent with the trial function space and can be used to compute the error between the exact solution $u$ and the numerical solution $u_{h}$ in $\Omega_{s}^{e}$. Although this norm is consistent with the mathematical theory by Leis [16] for the Helmholtz equation, it should only be used for the conjugated IEM, i.e. in the sesquilinear case. The stability and convergence analysis presented in $[2,13]$ show that the unconjugated IEM fails to converge in the far field, but converges rapidly in the near field. Therefore it does not make any sense to use the weighted $H^{1}$-norm to measure the error for the unconjugated IEM. The $L^{\infty}$-norm on the surface of the scatterer is used instead of the weighted $H^{1}$-norm in the bilinear formulation, which has also been used in $[2,13]$. In the bilinear formulations we compute

$$
\left\|u-u_{h}\right\|_{\infty}=\sup _{\boldsymbol{x} \in \Gamma}\left|u(\boldsymbol{x})-u_{h}(\boldsymbol{x})\right|,
$$

which is being done by replacing $\sup _{\boldsymbol{x} \in \Gamma}$ with the maximum over all Gausspoints for each element in $\Gamma$. In the sesquilinear formulations we meassure the error in the weighted $H^{1}$-norm, where

$$
\left\|u-u_{h}\right\|_{1, \Omega^{e}}^{2}=\left\|u-u_{h}\right\|_{1, \Omega_{s}^{e}}^{2}+\left\|u-u_{h}\right\|_{1, \Omega_{s}}^{2}
$$

with

$$
\left\|u-u_{h}\right\|_{1, \Omega_{s}^{e}}^{2}=\int_{\Omega_{s}^{e}} \frac{1}{r^{2}}\left|u-u_{h}\right|^{2} d \Omega_{s}^{e}+\int_{\Omega_{s}^{e}} \frac{1}{r^{2}}\left|\nabla\left(u-u_{h}\right)\right|^{2} d \Omega_{s}^{e}
$$

and

$$
\left\|u-u_{h}\right\|_{1, \Omega_{s}}^{2}=\int_{\Omega_{s}}\left|u-u_{h}\right|^{2} d \Omega_{s}+\int_{\Omega_{s}}\left|\nabla\left(u-u_{h}\right)\right|^{2} d \Omega_{s} .
$$

The rigid scattering of a plane wave on a sphere allows to determine the exact solution, which is the basis for a numerical convergence study. The exact solution $u$ for the rigid scattering on a sphere with radius 0.5 is given by (4.4) and the numerical solution in $\Omega_{s}^{e}$ can be represented in the form

$$
u_{h}(r, \boldsymbol{x})=\sum_{j=1}^{N} \sum_{i=1}^{M} u_{j i} N_{l(i, j)}(r, \boldsymbol{x}), \quad \boldsymbol{x} \in \Gamma_{s}, r>1,
$$

where $N_{l(i, j)}$ represent $h p$-basis functions of the IE and $u_{j i}$ denote the corresponding degrees of freedom. In $\Omega_{s}$ the numerical solution is given by

$$
u_{h}=\sum_{i=1}^{n r d o f} u_{i} \varphi_{i}
$$

where $\varphi_{i}, i=1, \ldots, n r d o f$, represents the $3 \mathrm{D}$ finite element shape functions. The evaluation of the error is done similar to the standard FE computations, for more details we refer to $[11,13]$. 


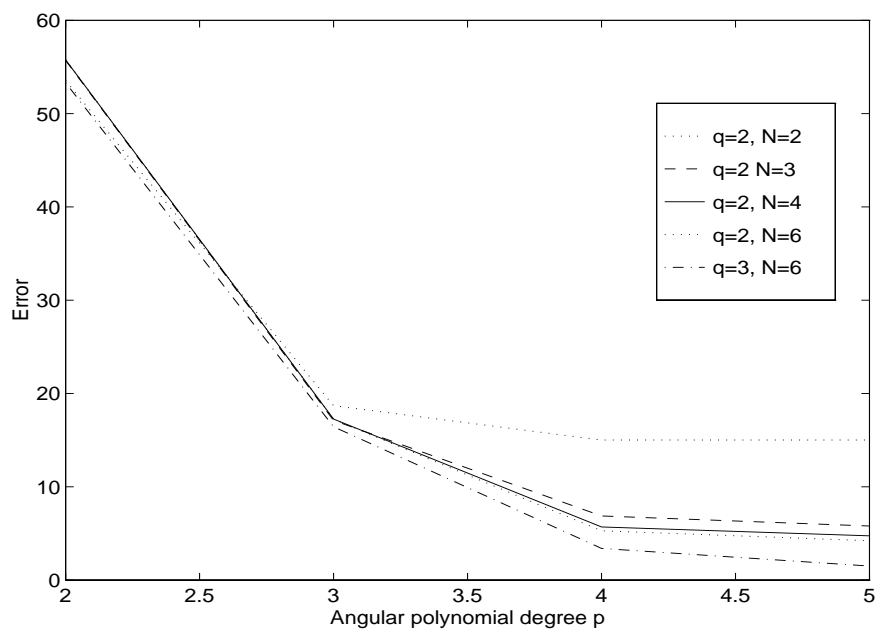

Figure 5: Scattering of a plane wave on a rigid sphere. Experimental p-convergence rates with $723 \mathrm{D}$ finite elements and 24 infinite elements with $k=10$.

\subsection{Numerical results}

In the following discussion we present numerical results for the sesquilinear Leis formulation for different examples of finite-infinite element meshes. It focuses on the question of, how the number of the shape functions in the radial direction affects the approximation of the exact solution.

The $p$-convergence rates are studied in terms of the weighted $H^{1}$-error norm on $\Omega^{e}$, compare (4.7). The error is analyzed in context of the order of approximation in the angular direction $p$ and the order of approximation $q$ in the radial direction. The influence of the number of IE shape functions $N$ on the numerical approximation becomes evident from the numerical results. In our numerical results we set the wave number $k$ to 10 and use up to 3 layers of $3 \mathrm{D}$ finite elements.

Figure 5 shows the $p$-convergence rates for different $q$ and number of radial shape functions respectively. An incident plane wave problem, described in section 4.2 , is assumed. This incident plane wave generates a scattered wave, which has an infinite number of terms in the radial direction. For the computations this series is truncated after 10 terms. The "y-axis" shows the error in percent of the weighted $H^{1}$-norm of the exact solution and the "x-axis" shows the order of approximation $p$.

These graphs clearly indicate that at a certain point it does not make any sense to increase the number of dof on the surface, unless the number of dof in radial direction is increased. Further, it is evident, that only enough dof in the angular and radial direction, e.g. high $p$ and $q$, or more finite elements, and the number of dof in radial direction of the infinite element sufficiently large does lead to a satisfactory result, i.e. the error 
with $p=5, q=3, N=6$ and three layers of finite elements is below $1.6 \%$, see Figure 5 . This clearly indicates that the discretization in the angular and the radial directions of the 3D finite elements is fine enough to obtain a reliable numerical solution.

It is also obvious that we do not observe the exponential shape of the $p$-convergence rates, as it might be expected. The reason for this behavior is that only one parameter, the polynomial degree in angular direction $p$, is varied, and that the quality of the approximation cannot be improved, if, for example, the discretization in the radial direction is not fine enough. In summary, Figure 5 clearly indicates, that $p$-, $q$ - and/or $h$-mesh refinements for the $3 \mathrm{D}-\mathrm{FE}$ mesh have to be performed simultaneously, along with a sufficient number of terms used in the radial direction of the infinite element.

Further, it is evident that the investigated finite-infinite element method works well for wave number $k=10$. The results show that the method is reliable, provided sufficiently fine meshes are used. We emphasize here that all computations are done on a workstation and that the numerical solution is obtained within fifteen minutes for fine meshes. This is a significant advantage compared to the execution time of standard boundary element formulations.

In Figure 6 we show the numerical solution for the rigid scattering on a finite cylinder with spherical incaps. The cylinder is assumed to be within the unit sphere and the Neumann boundary condition on the surface of the cylinder corresponds to an incident plane wave. The IEM is used to compute the numerical solution for the rigid scattering with wave number $k=10$. The finite element mesh contains three layers of finite elements with 216 finite elements per layer, $p=3$ and $q=2$. There are 216 infinite elements with $p=3$ and 6 radial shape functions. Altogether, 864 elements are used. The computation of the numerical solution with the coupled FE/IE methodology takes about twenty minutes on a workstation. We remark that the high demand of CPU time and memory makes it extremely difficult to obtain the numerical solution with the BEM for higher wave numbers. Figure 7 shows an adapted finite/infinite element mesh and indicates that the coupled finite/infinite element methodology can be incorporated into the standard adaptive mesh refinement technologies.

\section{Conclusions}

Different infinite element formulation for the exterior Helmholtz problem are analyzed. The presented methodologies apply to rigid scattering problems with general shape of the scatterers. The analyzed formulations combine a variable order $h p$-FE discretization between the scatterer and the unit sphere with an infinite element discretization on the boundary of the unit sphere. The infinite elements are obtained by combining $2 \mathrm{D} h p-$ FE shape functions with a spectral approximation in the third direction. The radial IE shape functions are motivated from the separation of variables approach. 


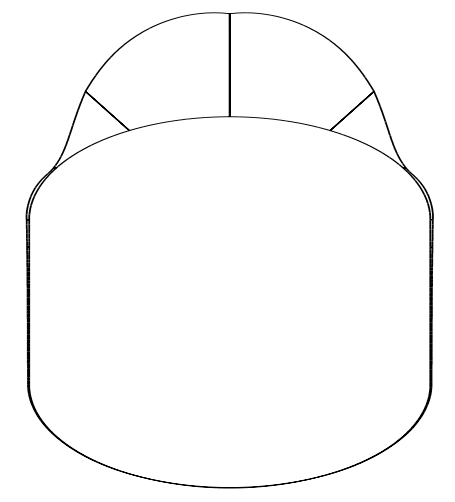

h-p

IEM/FEM

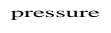

$\mathrm{k}=10.0$

absolute value

Figure 6: FEM/IEM. Scattering of a plane wave on a rigid cylinder with spherical incaps.

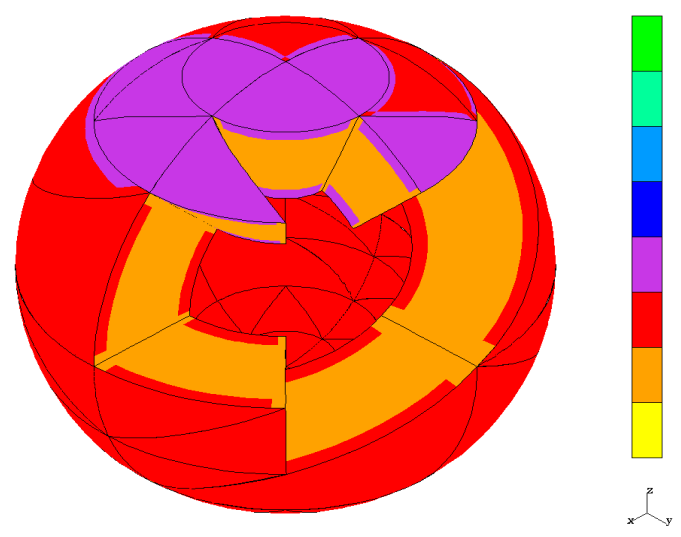

Figure 7: $h p$-adaptive finite-infinite element mesh. 
The theoretical results $[2,11,13]$ show that the method is reliable and computationally advantageous. The numerical results support this conclusion. The advantages and disadvantages of the conjugated and unconjugated formulations have already been pointed out in $[2,13]$ and the question for the optimal formulation is problem dependent, i.e. if only the near field solution is needed or if the solution is needed in the whole exterior domain.

\section{References}

[1] R. J. Astley, G. J. Macaulay and J. P. Coyette, "Mapped Wave Envelope Elements for Acoustical Radiation and Scattering", Journal of Sound and Vibration, vol. 170 , no. 1, pp. 97-118, 1994.

[2] I. Babuska and J. J. Shirron, "Solution of Exterior Helmholtz Problems using Finite and Infinite Elements", Dissertation preprint

[3] P. Bettess, Infinite Elements, Penshaw, Sunderland, UK, 1992.

[4] D. S. Burnett, “A Three-Dimensional Acoustic Infinite Element Based on a Prolate Spheroidal Multipole Expansion", Journal of the Acoustical Society of America, vol. 96 , pp. 2798-2816, 1994.

[5] L. Cremers, K. R. Fyfe and J. P. Coyette, "A Variable Order Infinite Acoustic Wave Envelope Element", Journal of Sound and Vibration, vol. 171, no. 4, pp. 483-508, 1994.

[6] L. Cremers and K. R. Fyfe, "On the use of Variable Order Infinite Wave Envelope Elements for Acoustic Radiation and Scattering", Journal of the Acoustical Society of America, vol. 97, no. 4, pp. 2028-2040, 1995.

[7] L. Demkowicz and K. Gerdes, "Convergence of the Infinite Element Methods for the Helmholtz Equation", to appear in Numerische Mathematik.

[8] L. Demkowicz and F. Ihlenburg, "Analysis of a Coupled Finite-Infinite Element Method for Exterior Helmholtz Problems”, TICAM Report 96-5D, 1996.

[9] L. Demkowicz and J. T. Oden, "Recent Progress on Application of $h p$-Adaptive BE/FE Methods to Elastic Scattering", International Journal for Numerical Methods in Engineering, vol. 37, pp. 2893-2910, 1994.

[10] K. Gerdes, L. Demkowicz, A. Bajer and C. Schwab, “A general 2D- $h p$-adaptive FE code based on triangular and quadrilateral elements", in preparation. 
[11] K. Gerdes and L. Demkowicz, "Solutions of 3D-Laplace and Helmholtz Equations in Exterior Domains using hp Infinite Elements", Comput. Methods Appl. Mech. Engrg. 137 (1996) 239-273.

[12] K. Gerdes, "Solution of the 3D Helmholtz equation in exterior domains of arbitrary shape using hp-finite infinite elements", SAM Report 96-D1, submitted to Finite Elements in Analysis and Design.

[13] K. Gerdes, "The conjugated vs. the unconjugated infinite element method for the Helmholtz equation in exterior domains", to appear in Computer Methods in Applied Mechanical Engineering.

[14] D. Givoli and J. B. Keller, "Special finite elements for use with high-order boundary conditions", Comp. Methods Appl. Mech. Engrg. 119 (1994), 199-213.

[15] A. Kirsch and P. Monk, "An analysis of the coupling of finite element and Nyström methods in acoustic scattering", IMA J. Num. Anal. (1994) 14, 523-544.

[16] R. Leis, Initial Boundary Value Problems in Mathematical Physics, Teubner, 1986.

[17] J. P. Wolf and C. Song, Finite-element modelling of unbounded media, Wiley, 1996. 